\title{
Does Telehealth Reduce Rural-Urban Care Access Disparities? Evidence from Covid-19 Telehealth Expansion
}

\author{
Shujing Sun \\ Naveen Jindal School of Management \\ University of Texas at Dallas \\ shujing.sun@utdallas.edu
}

\begin{abstract}
Using hospital claims data, we study the effect of telehealth expansion on the disparities between care access in rural and urban areas during Covid-19. We use urban areas as the control group and compare the changes in patients' access to care before and after the telehealth expansion. We find that the rural-urban disparities in overall access to care (i.e., the total number of visits) remain unchanged after the policy. We further distinguish in-person from telehealth visits and find enlarged disparities in patients' visiting modalities. In particular, urban patients substitute in-person visits with telehealth visits, yet rural patients have a much lower adoption rate of telehealth services and continue with in-person visits. Finally, we perform visit-level analyses and identify patients' social determinants and physicians' characteristics associated with telehealth adoptions.
\end{abstract}

\section{Introduction}

Rural areas of the U.S. have about $20 \%$ of the population but less than $10 \%$ of physicians 1 The average travel distance from a patient to a physician is 10.5 miles in rural areas compared with 4.4 miles in urban areas 2 The combination of a low physician-patient ratio and long travel distance has impeded rural patients' access to health care and, consequently, led to poor health outcomes. Studies have found rural Americans are worse in health conditions than their urban counterparts and more likely to die from heart diseases, cancers, and chronic lower respiratory diseases $3^{3}$ To reduce the rural-urban disparities in care access, the federal and state governments have implemented various policies to improve health care access in rural areas. For example, many states have expanded Medicaid to low-income adults. These

${ }^{1}$ See details at https://www.census.gov/programs-surveys/

2 https://www.pewresearch.org/fact-tank/2018/12/12/

${ }^{3}$ https://www.cdc.gov/ruralhealth/about.html

\author{
Guihua Wang \\ Naveen Jindal School of Management \\ University of Texas at Dallas \\ guihua.wang@utdallas.edu
}

expansions are critical to rural hospitals that are on the verge of closing and heavily relying on Medicaid reimbursements for health care services. To ease the shortage of physicians, a number of states have established legislation that expands the roles and responsibilities of non-physician primary care providers - such as nurse practitioners and physician assistants - when they practice in medically underserved communities such as rural areas 4

With advances in telehealth platforms and remote patient monitoring technologies, researchers and policymakers proposed using telehealth to reduce disparities in care between rural and urban areas. Telehealth has the potential to improve rural Americans' access to care for two reasons. First, it significantly reduces or even eliminates traveling costs, so patients can visit health care providers whenever needed. Second, patients have more options when choosing physicians, as telehealth allows them to visit faraway health care providers via virtual technologies. Despite its great promise, several barriers limit the extensive use of telehealth. First, physicians and patients were reluctant to use telehealth due to lower reimbursement rates, difficulty in establishing patient-provider relationships, and legal issues such as state licensure laws [1]. Second, according to CDC, patients of rural areas in the United States tend to be older than their urban counterparts ${ }^{5}$ Consequently, rural patients may be unwilling to use telehealth due to a lack of digital literacy. Besides, telehealth requires high-speed internet access and smartphones or related technologies. Rural Americans without these assets will not be able to use telehealth even if they are willing to visit health care providers virtually. Probably because of these barriers, the percentage of health care providers adopting telehealth is only $22 \%$ in $2019{ }^{6}$

While the use of telehealth was limited before 2020, the outbreak of Covid-19 has significantly propelled

${ }^{4}$ https://www.ncsl.org/research/health/improving-rural-health-state-policy

${ }^{5}$ https://www.cdc.gov/ruralhealth/about.html

${ }^{6}$ https://www.beckershospitalreview.com 
the adoption of telehealth services. The pandemic has blocked patients' access to their primary care physicians and hospitals. For those who manage to have an in-person visit, the exposure may significantly increase the risks of Covid-19 infection for both the patients and their health care providers. Telehealth can help reduce the impact of Covid-19 while ensuring patients having access to care. First, because telehealth allows patients to visit health care providers remotely, it reduces person-to-person contacts and the spread of the virus [2]. Second, because telehealth minimizes the inconvenience of traveling, it allows patients to visit faraway health care providers when nearby providers face capacity issues. Recognizing the great potential of telehealth, policymakers called for an increase in access to telehealth services. Following the initiative, many payers had expanded the coverage of telehealth visits. For example, the Blue Cross and Blue Shield extended the coverage for telehealth services on March 19, 2020, by waiving cost-sharing for telehealth services for fully insured members and advocating for physician and health system adoption of social distancing-encouraged capabilities ${ }^{7}$

Despite the clear benefits of telehealth in containing the Covid-19 pandemic, it is unclear how the telehealth expansion affects care access and whether such effects might differ by rural and urban areas. More specifically, we aim to answer three important questions: (1) Does the expansion reduce the disparity of total care access in rural and urban areas? (2) How does the expansion change patients' visiting modalities (i.e., telehealth or in-person visits) in rural and urban areas? (3) What are some of the barriers that prevent health care providers and patients from adopting telehealth? Towards this goal, we collect national claims data of a large private health insurance provider that implemented the telehealth expansion policy. This payer expanded its telehealth coverage in March 2020. Before the telehealth expansion, patients have limited reimbursement for telehealth services. After the telehealth expansion policy, all members across the U.S. have access to an expanded list of procedures and services that are reimbursable when delivered via telehealth.

The main challenge to addressing these questions is that the telehealth expansion applies to patients of all areas around the same time. As a result, we cannot use a difference-in-differences (DID) approach to compare areas that did not expand telehealth with those that did to analyze the effect of telehealth expansion. We address this challenge by using urban counties as the "control" group and compare the differential treatment effects between rural and urban counties before and after the expansion. This approach has been used in several other studies. For example, [3] used male researchers as a control group to study the effect of Covid-19 (which applies to both male and female researchers) on gender inequality in research productivity. Another challenge is that telehealth expansion was introduced during Covid-19. Any changes in disparities between care in rural and urban areas may be due to Covid-19 instead of telehealth expansion. To alleviate this concern, we conduct analyses with and without controlling for the Covid-19 infections and compare whether the results from these two models are significantly different from each other. The third challenge is that states implemented several other policies to contain the Covid-19 pandemic. For example, some states implemented stay-at-home orders, requiring residents to stay at home except for essential trips. A few other states issued guidance to suspend non-essential surgery in an effort to save limited health care resources for Covid-19 patients. There might be a concern that changes in disparities may be driven by these statewide policies. We address this concern by controlling a battery of Covid-19 related policies that might affect patients' way of accessing care and were implemented during our sample period.

The findings suggest that rural-urban disparities in patients' overall access to care have not been much affected by the telehealth expansion policy. Interestingly, the composition of patients' visiting modalities changed dramatically due to the telehealth expansion policy. In particular, while the total number of visits from rural and urban areas follow a similar trend, urban patients' in-person visits are significantly reduced. Yet, rural patients' in-person visits are not much affected. In other words, urban patients substitute in-person visits with telehealth visits. In contrast, rural patients have a much lower adoption rate of telehealth services and continue with in-person visits. Given that telehealth is preferred over in-person visits during the pandemic, we conclude that the disparities in the way of accessing care significantly increased. We supplement the paper with visit-level analyses and identify patients' social determinants and physicians' characteristics that might have affected telehealth adoptions. As telehealth is likely to become an integral part of healthcare delivery, we believe our findings are important to researchers and providers in understanding the implications of telehealth expansion on rural-urban disparities and the existing barriers when expanding telehealth in rural areas.

${ }^{7}$ https://www.bcbs.com/press-releases/media-statement 


\section{Literature Review}

Our study relates to two streams of literature: (1) use of telehealth and (2) rural-urban disparity. We briefly review these two streams of literature.

\subsection{Use of Telehealth}

There are several recent publications investigating the applications and impacts of telehealth in several clinical settings. From the perspective of patients, [4] conducted a randomized controlled clinical trial on twenty patients with Parkinson's disease. The paper finds that web-based videoconferencing to provide specialty care at home is feasible, provides value to patients, and may offer similar clinical benefits to that of in-person care. [5] studied the impact of e-visits on visit frequencies and patient health and found e-visit adoption increases the number of office visits and reduces the acceptance of new patients. [6] studied the impact of telehealth centers in Southern India and found the opening of telehealth centers increases the overall network visit rate but reduces hospital visits, which suggests patients substitute hospital visits with telehealth center visits.

From the perspective of hospital operations and physicians' workload management, [7] developed a partially observable Markov process to study the effectiveness of telemedical physician triage in workload management. The authors found lower-level agents should make decisions on a higher proportion of cases as the workload at the upper level increases. [8] developed a Markov decision process to study the effectiveness of teletriage in managing healthcare demand. The authors found the addition of teletriage increases the rate of arrivals to the emergency department. [9] developed a game-theoretic model to compare the strategic behavior of revenue-maximizing and welfare-maximizing specialists. The authors found the latter serve a large patient population, spend less time with patients, and have shorter waiting times. [10] studied the impact of telemedicine on emergency room congestion. The paper found that telehealth availability significantly reduces emergency patients' waiting time and length of stay through flexible resource allocation.

\subsection{Rural-Urban Disparity}

The rural-urban disparity in healthcare access has been a prevalent issue and has drawn increasing attention to researchers worldwide. Most of the existing studies compared the health of residents and access to providers in the U.S. For example, [11] studied associations between place-based characteristics of rural-urban status and health among adults above 65 and found an increase in urbanicity correlates with a decrease in negative self-reported health. [12] studied disparities in health care provider availability in rural versus urban Alaska and New Mexico and found rural residents have significantly less access to health care providers, and discrepancies increase with the level of required provider education and specialization. A number of studies examined health disparities in other countries and obtained similar results. For example, [13] analyzed patterns in physician and hospital utilization among rural and urban populations in China and found rural residents use physicians more than urban residents and use hospitals less. [14] studied regional differences between rural and urban areas in the management of acute myocardial infarction (AMI) in Japan and found AMI patients in rural areas are less likely to be transported directly to facilities with percutaneous coronary interventions.

With the advancement in information technology, researchers have found the potential of alleviating the healthcare gap using technology-mediated healthcare platforms and systems. For instance, [15] studied the social value of an online health community and found urban users are net suppliers of social support while rural participants are net recipients, which suggests that online health communities can help alleviate rural-urban health disparities. [16] studied the effect of telemedicine on access to acute stroke care for racial and ethnic minorities in Texas. The paper found that telemedicine increased access to stroke expertise and did not find evidence of disparities in access to acute stroke expertise via telemedicine. [17] studied the effect of a virtual urgent care program on health equity and found the program reduced the disparity between rural and urban residents. [18] studied the use of telemedicine in a large healthcare system in New York City and found black patients are less likely to use telemedicine than white patients.

Compared to the existing literature, our paper has several notable contributions. First, the literature has recognized the potential of telemedicine, yet the adoption of telemedicine has not reached its full extent due to the lack of knowledge of its general implications [10]. Utilizing the telehealth expansion and the subsequent spike in telehealth adoption during the Covid-19 pandemic, we examined the causal impact of telehealth expansion on rural-urban disparity. Second, taking advantage of the unique large-scale data with individual's visiting modality (i.e., telehealth vs. in-person visit), we identify potential barriers to telehealth adoption and the effect of telehealth adoption on reducing Covid-19 infection. Our findings are critical 
to researchers and policymakers, as telehealth is likely to become an integral part of healthcare delivery, even beyond the pandemic.

\section{Research Background and Data Description}

The global spread of Covid-19 is an unprecedented and unexpected shock to the world. Following the national emergency declaration on March 13, 2020, the government, providers, payers, and healthcare researchers have exerted relentless efforts to combat the Covid-19 outbreak. Among various initiatives, telehealth expansion has become a centerpiece that ensures patients' access to care while reducing the risks of Covid-19 exposure. For example, the Blue Cross Blue Shield Association, which provides health insurance to more than 106 million people in the United States, announced the coverage of telehealth services for its members on March 19, 20208 8 Our paper aims to examine the impact of such telehealth expansion policy on patients' access to care and rural-urban care access disparities.

Our primary sample comprises individual patient claims to an anonymous payer, and the sample period ranges from October 2019 to July 2020. This anonymous payer is one of the largest private health insurance providers, serving over 100 million people across the U.S. For each claim, we have information on the date of service, patient's demographic information (e.g., age, gender, and race), patient's diagnosis code, Current Procedural Terminology (CPT) code, and Healthcare Common Procedure Coding System (HCPCS) code, and the information of physicians who provided the care.

This payer expanded its telehealth coverage in March 2020. Before the telehealth expansion policy, patients have limited reimbursement for telehealth services. After the telehealth expansion policy, the payer waived cost-sharing for all members across the U.S. over an expanded list of procedures and services that are delivered via telehealth by in-network providers. More specifically, the anonymous payer in our sample covers telehealth codes consistent with the permanent code lists from the CMS-specified list ${ }^{9}$ The CMS list covers a wide range of services, ranging from real-time telehealth visits between providers and patients, patient-initiated virtual check-in, and e-visits between patients and their providers through an online patient portal. The expanded list also covers services for various diagnoses, such as psychotherapy,

${ }^{8}$ https://www.bcbs.com/press-releases

s https://www.cms.gov/Medicare/ ophthalmological services, care for chronic diseases (e.g., diabetes, cardiac rehabilitation, and hypertension), general health and behavioral intervention, etc.

As we aim to investigate how telehealth expansion affects patients' access to care and visit modality, we restrict the sample to visits with procedures and services that are eligible for reimbursement under the CMS list, because patients associate with these visits are the direct beneficiaries of the telehealth expansion policy. We distinguish in-person from telehealth visits based on the modifiers appended to the HCPCS or CPT code for each claim. Specifically, claims with one of the following modifiers (G0, GT, GQ, and 95) are furnished via telehealth 10 The modifier G0 identifies telehealth services for diagnosis, evaluation, or treatment of symptoms of an acute stroke. The modifier GT is the most commonly used modifier for telehealth claims, which recognizes telehealth services via interactive audio and video telecommunications systems. The modifier GQ identifies asynchronous telehealth services. The modifier 95 is a fairly new modifier and is used only when billing to private payers to indicate that services were rendered via synchronous telecommunication.

To account for confounding factors related to the Covid-19 situation and policies, we supplement the main dataset with the county-level Covid-19 cases from the Johns Hopkins University ${ }^{11}$ and the state-level policies from the Kaiser Family Foundation $(\mathrm{KFF})^{12}$ In the propensity score matching, we further collect county-level characteristics from the 2018 CDC Social Vulnerability Index, which contains 2014-2018 American Community Survey (ACS) estimates for various demographic and socioeconomic variables ${ }^{13}$

\section{Econometric Model}

In this section, we first illustrate the empirical challenges and our identification strategy. We then describe the difference-in-differences (DID) model.

\subsection{Empirical Strategy}

The main challenge we face in analyzing the impact of telehealth expansion is that all counties across the U.S. experienced the same expansion simultaneously, which makes it infeasible to directly apply the DID approach. Following the existing literature [19, 3], we extend the DID framework by taking rural areas as the treatment group and urban areas as the control group.

\footnotetext{
10 https://www.bcbstx.com/provider/pdf

${ }^{11}$ https://coronavirus.jhu.edu/us-map

12 https://www.kff.org/report-section/

${ }^{13}$ https://www.atsdr.cdc.gov/placeandhealth/
} 
Note that although the telehealth expansion applies to all patients, regardless of their rural or urban residences, the DID framework allows estimating the treatment effects by comparing the differences between rural and urban areas before and after the telehealth expansion.

The validity of this approach depends on two assumptions. The first is the parallel trends assumption, which is the most critical assumption. It requires that the outcome difference between the rural and urban areas stays constant in the absence of the expansion. The second assumption is that the expansion is exogenous to patients and providers. In other words, they did not anticipate the expansion, and hence there were no strategic behaviors in deciding the modality of their clinical visits before the implementation of telehealth expansion.

We test the violation of the first assumption by including pre-treatment dummies as independent variables following the literature [3]. Although there is no empirical way to test the second assumption, it is likely to be valid. Because Covid-19 was an unexpected shock, and the payer in our sample is one of the first to respond to the pandemic by delivering its telehealth expansion initiative, patients and providers were unlikely to foresee the policy change and adjust their behaviors ex-ante.

\subsection{Difference-in-differences Model}

We arrange the data at the county-month level and the specification is as follows:

$$
\begin{aligned}
& Y_{i t}=\alpha_{0}+\alpha_{1} \text { Rural }_{i} \times \text { TeleExpansion }_{t} \\
& +\beta X_{i t}+\text { CountyF } E_{i}+\text { TimeF } E_{t}+\varepsilon_{i t} \text {. }
\end{aligned}
$$

where $Y_{i, t}$ corresponds to the log-transformed number of hospital visits in county $i$ at month $t$. We log-transform the dependent variable due to its skewed distribution. The transformation also facilitates the interpretation and comparison of coefficient estimates across different counties 14 Since our first research question is on health care access disparity, we construct the first dependent variable as $\ln$ (TotalVisits $\left._{i, t}\right)$, which represents the total number of visits in county $i$ at month $t$. To further examine changes in patients' visiting modalities, we then construct $\ln \left(\right.$ In-personVisits $\left.{ }_{i, t}\right)$ and $\ln \left(\right.$ TeleVisits $\left._{i, t}\right)$, which represents the log-transformed number of in-person and telehealth visits in county $i$ at month $t$, respectively. Following the 2013 NCHS Urban-Rural Classification Scheme, we define a county

\footnotetext{
${ }^{14}$ The results remain qualitatively the same when we construct the dependent variable in its original scale. Results are available upon request.
}

as rural (i.e., Rural $_{i}=1$ ) if it is classified as "micropolitan" or "non-core" 15 The binary variable

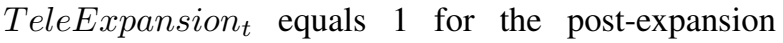
period (i.e., starting March, 2020).

The coefficient estimate $\alpha_{1}$ of the interaction term captures the treatment effect of telehealth expansion on rural areas relative to urban areas. As we have accounted for the county and time fixed effects, the main effect of Rural $_{i}$ is absorbed in the county

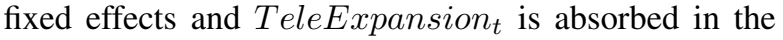
time fixed effects. In robustness checks, we further control for time-varying characteristics $X_{i t}$, such as county-level Covid-19 infections and the state policies. Throughout the analyses, we cluster the standard errors at the state level to account for possible unobserved correlations in error terms within each state. Given that a stable composition of treatment and control groups pre- and post- telehealth expansion is critical for unbiased estimation of the DID effects [20], we dropped counties with $20 \%$ or more missing observations at the monthly level and expand the panel to be balanced. Our motivation is to keep as many valid observations as possible while excluding outliers (i.e., counties with no patient visits at all at the monthly level).

\section{Main Results}

In this section, we first estimate the main effects of telehealth expansion on total visits. We then decompose the total visits by visiting types and examine changes in patients' visiting modalities.

\subsection{Effect on Total Visits}

Before presenting the DID results, we begin with checking the model-free summary statistics on overall access (i.e., total hospital visits) by patients' residences. Before the expansion, the average number of visits is 70.34 in rural areas and 760.43 in urban areas, so the difference before the expansion is $-687.39(=73.04-$ 760.43). After the expansion, the number of visits is 102.78 in rural areas and 1084.64 in urban areas, so the difference after the expansion is $-981.86(=$ $102.78-1084.64)$. The model-free DID estimate is thus $-294.47(=-981.86-(-687.39))$, suggesting a relative decrease in the volume of total visits of rural patients relative to urban patients. Note that this model-free analysis does not control for county and time fixed effects or time-varying county characteristics.

To formally analyze the effect of telehealth expansion on patients' total access to health care, we execute the DID regression model 1 1 in Section

\footnotetext{
${ }^{15}$ https://www.cdc.gov/nchs/data_access/urban_rural.htm
} 
Table 1. Effect of Telehealth Expansion on Total Visits

\begin{tabular}{lccc}
\hline \multirow{2}{*}{ Variable } & \multicolumn{3}{c}{$\ln$ (TotalVisits) } \\
\cline { 2 - 4 } & $(1)$ & $(2)$ & $(3)$ \\
\hline Rural $\times$ TeleExpansion & -0.023 & -0.015 & -0.0004 \\
& $(0.026)$ & $(0.026)$ & $(0.026)$ \\
County \& Time FE & Y & Y & Y \\
Covid Cases & $\mathrm{N}$ & $\mathrm{Y}$ & $\mathrm{Y}$ \\
State Policy & $\mathrm{N}$ & $\mathrm{N}$ & $\mathrm{Y}$ \\
\hline Observations & 13,280 & 13,280 & 13,280 \\
R-Squared & 0.433 & 0.433 & 0.437 \\
\hline
\end{tabular}

${ }^{*} p<0.1,{ }^{* *} p<0.05,{ }^{* * *} p<0.01$

Econometric Model. The results are reported in Table 1 In column (1), we observe a negative but insignificant treatment effect of the telehealth expansion policy, implying that the total number of visits evolved in parallel patterns and remains similar for rural and urban areas. The results remain robust when imposing additional controls that might affect rural and urban patients' need for hospital visits and correlate with the policy expansion, such as county-level Covid cases (column (2)) and state-specific stay-at-home orders (column (3)). Both estimates are small and insignificant, which indicates that rural-urban care access disparity in overall access is not significantly affected. Hence, the answer to the first question in the Introduction is no. The telehealth expansion policy does not reduce rural-urban care access disparities in our sample.

\subsection{Change in Visit Modality}

To address the second question regarding patients' choices of visiting modality, we now turn to analyze the change in the number of visits by modality. Before the expansion, the average number of in-person visits is 71.67 in rural areas and 742.68 in urban areas, so the difference before the expansion is $-671.01(=$ $71.67-742.68)$. After the expansion, the number of in-person visits is 72.71 in rural areas and 587.42 in urban areas, so the difference after the expansion is $-514.71(=72.71-587.42)$. The model-free DID estimate is thus $156.30(=-514.71-(-671.01))$. In other words, urban patients significantly reduced their in-person visits following the telehealth expansion. In contrast, rural patients' in-person visits did not change much. Regarding telehealth visits, we observe an increase in both rural and urban areas. However, the magnitude of increase is much larger in urban areas. The average number of telehealth visits increases from 1.37 to 30.07 in rural areas, and from 17.74 to 497.22 in urban areas. Therefore, the non-parametric DID estimate for telehealth visits is $-450.78(=-467.15-(-16.37))$.
Table 2. Effect of Telehealth Expansion on In-person and Telehealth Visits

\begin{tabular}{lccc}
\hline & \multicolumn{3}{c}{$\ln ($ In-personVisits $)$} \\
& $(1)$ & $(2)$ & $(3)$ \\
\hline Rural $\times$ TeleExpansion & $0.125^{* * *}$ & $0.126^{* * *}$ & $0.133^{* * *}$ \\
& $(0.042)$ & $(0.042)$ & $(0.041)$ \\
County \& Month FE & $\mathrm{Y}$ & $\mathrm{Y}$ & $\mathrm{Y}$ \\
Covid Cases & $\mathrm{N}$ & $\mathrm{Y}$ & $\mathrm{Y}$ \\
State Policy & $\mathrm{N}$ & $\mathrm{N}$ & $\mathrm{Y}$ \\
\hline Observations & 13,280 & 13,280 & 13,280 \\
R-Squared & 0.3484 & 0.3492 & 0.3499 \\
\hline & \multicolumn{3}{c}{$\ln ($ TeleVisits $)$} \\
& $(4)$ & $(5)$ & $(6)$ \\
\hline Rural $\times$ TeleExpansion & $-1.716^{* * *}$ & $-1.627 * * *$ & $-1.583^{* * *}$ \\
& $(0.143)$ & $(0.143)$ & $(0.141)$ \\
County \& Month FE & $\mathrm{Y}$ & $\mathrm{Y}$ & $\mathrm{Y}$ \\
Covid Cases & $\mathrm{N}$ & $\mathrm{Y}$ & $\mathrm{Y}$ \\
State Policy & $\mathrm{N}$ & $\mathrm{N}$ & $\mathrm{Y}$ \\
\hline Observations & 13,280 & 13,280 & 13,280 \\
R-Squared & 0.6457 & 0.6516 & 0.6552 \\
\hline
\end{tabular}

Following the regression model 1 , we replicate the DID analysis by constructing the dependent variables as the log-transformed number of in-person and telehealth visits, respectively. The results are reported in Table 2 From columns 1-3, we observe a significant and positive treatment effect on rural patients. This implies that patients in rural areas have significantly greater tendencies to have in-person visits relative to those of urban areas. In terms of magnitude, compared to urban counties, rural counties experienced a $13.3 \%$ increase (see column 3) in in-person visits. From columns 4-6, we observe a significantly negative effect on telehealth visits, suggesting that rural areas are less likely to adopt telehealth than urban areas. Take column 6 as an example, rural patients' volume of telehealth visits is $158.3 \%$ lower than that of urban patients after the policy change. Overall, the findings show that urban patients substitute in-person visits with telehealth visits, but rural patients continue with the in-person visits.

\section{Robustness Checks}

To further evaluate the validity of our main empirical findings, we start with a pre-trend analysis to test if there is any violation of the parallel trend assumption. We then conduct a set of tests to account for various other state-level policies related to Covid-19. We conclude with propensity score matching, which enables us to construct a more comparable sample of rural and urban counties. 


\subsection{Pre-trend Analysis}

A fundamental identification assumption in the DID model is the parallel trend assumption. To evaluate whether there are any signs of violation for this assumption, we create a series of lag indicators for each pre-treatment period and two lead indicators for the time periods following the telehealth expansion. More specifically, we expand the regression model 1 as follows:

$$
\begin{aligned}
& Y_{i t}=\alpha_{0}+\sum_{\tau=1}^{5} \alpha_{t-\tau} \text { Rural }_{i} \times \text { Time }_{t-\tau} \\
& +\alpha_{t=0} \text { Rural }_{i} \times \text { Time }_{t=0}+\alpha_{t \geq 1} \text { Rural }_{i} \times \text { Time }_{t \geq 1} \\
& +\beta X_{i t}+\text { CountyF }_{i}+\text { TimeF }_{t}+\varepsilon_{i t}
\end{aligned}
$$

where $Y$ can be $\ln$ (TotalVisits) or $\ln$ (In-personVisits) ${ }^{16}$ The coefficient estimates $\left(\alpha_{t-4}, \ldots, \alpha_{t-1}\right)$ capture the month-by-month pre-treatment differences between rural and urban areas. $\alpha_{t=0}$ and $\alpha_{t \geq 1}$ stand for the DID effect in the month and following months of telehealth expansion, respectively. The results are reported in Table 3 , where the baseline period is 5-month before the expansion (i.e., 2019 October). We find no evidence of pre-trend differences in total visits and in-person visits between rural and urban areas, because the coefficient estimates for all the lag indicators are small and insignificant. We can see that the divergence of in-person visits between rural and urban areas emerged only since the policy implementation month.

\subsection{State-level Policy}

Besides the stay-at-home orders, some states also issued guidance to suspend non-essential surgery to preserve limited healthcare resources for Covid-19 patients. Despite that this initiative is to curb non-essential procedures, it has been shown to also hurt the availability of essential ones [21]. Similarly, other state policies, such as surgery resumption and state reopen, may also affect patients' visiting modalities directly or indirectly. To check if these state-level policies drive the heterogeneous changes in patients' visiting behaviors between rural and urban areas, we replicate the DID analysis (equation 11) with additional controls of three other state-level policies that were implemented during our sample period and might affect patients' modalities to access health care. In particular, besides the stay-at-home orders that were implemented

\footnotetext{
${ }^{16}$ Note that we do not need to test the pre-trend of $\ln$ (Televisits) because there were zero telehealth visits for most counties before the telehealth expansion. In other words, the parallel trend automatically satisfies between rural and urban areas.
}

Table 3. Pre-trend Analysis

\begin{tabular}{lcc}
\hline & $\begin{array}{c}\ln (\text { TotalVisits) } \\
(1)\end{array}$ & $\begin{array}{c}\ln (\text { In-personVisits }) \\
(2)\end{array}$ \\
\hline Rural $\times$ Time $_{t-4}$ & 0.036 & 0.037 \\
& $(0.027)$ & $(0.027)$ \\
Rural $\times$ Time $_{t-3}$ & 0.026 & 0.025 \\
& $(0.025)$ & $(0.026)$ \\
Rural $\times$ Time $_{t-2}$ & 0.029 & 0.031 \\
& $(0.034)$ & $(0.034)$ \\
Rural $\times$ Time $_{t-1}$ & 0.015 & 0.015 \\
& $(0.041)$ & $(0.041)$ \\
Rural $\times$ Time $_{t=0}$ & 0.053 & $0.090^{* * *}$ \\
& $(0.044)$ & $(0.045)$ \\
Rural $\times$ Time $_{t \geq 1}$ & 0.027 & $0.166^{* * *}$ \\
& $(0.034)$ & $(0.048)$ \\
County \& Month FE & $\mathrm{Y}$ & $\mathrm{Y}$ \\
Covid Cases & $\mathrm{Y}$ & $\mathrm{Y}$ \\
State Policy & $\mathrm{Y}$ & $\mathrm{Y}$ \\
\hline Observations & 13,280 & 13,280 \\
R-Squared & 0.4367 & 0.3502 \\
\hline
\end{tabular}

${ }^{*} p<0.1,{ }^{* *} p<0.05,{ }^{* * *} p<0.01$

around the same time as the telehealth expansion policy, we further control for policies regarding the suspension of elective medical procedures, the resumption of elective medical procedures, and the reopen $\sqrt{17}$ Since the policy implementation date differs by state, we generate binary variables to indicate the post-policy periods of different policies based on a patient's location. Table 4 reports the regression results using different dependent variables. We find consistently robust results, suggesting that our findings are not driven by these confounding policy implementations.

Table 4. Control Additional Covid-Related Policies

\begin{tabular}{lccc}
\hline & $\begin{array}{c}\ln (\text { TotalVisits) } \\
(1)\end{array}$ & $\begin{array}{c}\ln \text { (In-personVisits) } \\
(2)\end{array}$ & $\begin{array}{c}\ln \text { (TeleVisits) } \\
(3)\end{array}$ \\
\hline Rural $\times$ TeleExpansion & -0.001 & $0.131^{* * *}$ & $-1.582^{* * *}$ \\
& $(0.027)$ & $(0.040)$ & $(0.140)$ \\
Stay-at-home Order & $0.181^{* *}$ & 0.085 & $0.504 * *$ \\
& $(0.071)$ & $(0.091)$ & $(0.235)$ \\
Surgery Suspension & -0.057 & -0.035 & -0.055 \\
& $(0.044)$ & $(0.073)$ & $(0.163)$ \\
Surgery Resumption & $0.081^{* * *}$ & 0.042 & 0.052 \\
& $(0.023)$ & $(0.048)$ & $(0.102)$ \\
State Reopen & -0.030 & 0.065 & -0.174 \\
& $(0.027)$ & $(0.061)$ & $(0.13)$ \\
County \& Month FE & $\mathrm{Y}$ & $\mathrm{Y}$ & $\mathrm{Y}$ \\
Covid Cases & $\mathrm{Y}$ & $\mathrm{Y}$ & $\mathrm{Y}$ \\
State Policy & $\mathrm{Y}$ & $\mathrm{Y}$ & $\mathrm{Y}$ \\
\hline Observations & 13,280 & 13,280 & 13,280 \\
R-Squared & 0.437 & 0.350 & 0.655 \\
\hline${ }^{*} p<0.1,{ }^{* *} p<0.05,{ }^{* * *} p<0.01$ & & &
\end{tabular}

\subsection{Propensity Score Matching}

Despite a wide range of controls, one may still worry that the finding could be driven by the selection bias between rural and urban patients. For example,

\footnotetext{
${ }^{17}$ https://www.mcguirewoods.com/client-resources/Alerts/2020/10/state-governors-stay-a
} 
rural areas may have lower patient volume to hospitals, therefore lower exposure risks of in-person visits. Accordingly, rural patients may be less worried about going to the doctor's office and continue with in-person visits, regardless of telehealth expansion. To further alleviate this concern, we refer to propensity score matching (PSM) [22]. The idea is to reduce selection bias by making the treatment and control groups (i.e., rural and urban counties) more comparable with respect to the pre-treatment covariates.

Table 5. Impact of Telehealth Expansion on Hospital Visits by Types - PSM

\begin{tabular}{lccc}
\hline & $\begin{array}{c}\ln (\text { TotalVisits) } \\
(1)\end{array}$ & $\begin{array}{c}\ln (\text { In-personVisits) } \\
(2)\end{array}$ & $\begin{array}{c}\ln \text { (TeleVisits) } \\
(3)\end{array}$ \\
\hline Rural $\times$ TeleExpansion & 0.009 & $0.153 * *$ & $-0.497 * * *$ \\
& $(0.046)$ & $(0.069)$ & $(0.151)$ \\
County, Month FE & $\mathrm{Y}$ & $\mathrm{Y}$ & $\mathrm{Y}$ \\
Covid Cases & $\mathrm{Y}$ & $\mathrm{Y}$ & $\mathrm{Y}$ \\
State Policy & $\mathrm{Y}$ & $\mathrm{Y}$ & $\mathrm{Y}$ \\
\hline Observations & 7,080 & 7,080 & 7,080 \\
R-Squared & 0.346 & 0.276 & 0.449 \\
\hline${ }^{*} p<0.1,{ }^{* *} p<0.05,{ }^{* * *} p<0.01$ & & &
\end{tabular}

To implement the PSM, we start with a cross-sectional sample of rural and urban counties, and match counties based on various characteristics before telehealth expansion. More specifically, we match by the 3-month historical visits (i.e., from 2019 July to 2019 September) so that the matched counties have similar patient volumes before the policy change. We also include a wide range of variables from the 2018 CDC Social Vulnerability Index in the matching, including healthcare access, socioeconomic characters, household senior and disability composition, minority status, and transportation condition. Follow the literature [23], we allow for replacement in the matching since there are much fewer control counties (i.e., urban areas) that are comparable to the treatment counties (i.e., rural areas) in variables such as patient volume and population size. Using propensity score matching with a caliper of 0.01 and allowing for replacement, we identify 708 matched counties (542 rural counties and 166 urban counties) 18 The matched sample is balanced in all matching variables. Table 5 reports the regression results. All results remain robust and are consistent with our main findings.

\section{Discussion and Insights}

In this section, we first identify some of the barriers that prevent healthcare providers and patients from adopting telehealth. We then analyze whether telehealth adoption reduces Covid-19 infections.

\footnotetext{
${ }^{18}$ Note that the result of propensity score matching is not sensitive to the threshold of calipers 0.05 or 0.1 . Results corresponding to different parameter setups are available upon request.
}

\subsection{Barriers to Telehealth Adoption}

We now address the third question from the Introduction by performing visit-level analyses and examining patients' social determinants and physicians' characteristics that might have affected their adoptions of telehealth.

From the patient side, we consider how social determinants associate with one's telehealth adoption. We start with extracting patient visits to only providers who adopted telehealth into their practice. For example, if a provider conducted her first telehealth visit on April 3, 2020, we define this provider as an adopter and include only clinical visits to this provider after April 3, 2020. The rationale is that given a provider is an adopter, the modality for a visit depends more on a patient's choice. Using the visit-level data in this subsample, we conduct a logistic regression and report the results in column 1 of Table 6. We can see that patients from rural areas, patients above 65 , and male patients are less likely to use telehealth. Consistent with the telehealth initiative to overcome the geographic barriers, we observe that patients who are in different locations from providers (i.e., patients and providers do not share the same zip code) are more likely to utilize telehealth.

Table 6. Barriers to Telehealth Adoption

\begin{tabular}{lcc}
\hline \multirow{2}{*}{ Variable } & \multicolumn{2}{c}{ Telehealth Adoption (Binary) } \\
\cline { 2 - 3 } & $\begin{array}{c}\text { Patient } \\
(1)\end{array}$ & $\begin{array}{c}\text { Provider } \\
(2)\end{array}$ \\
\hline Rural & $-0.576^{* * *}$ & \\
Age Above 65 & $(0.006)$ & \\
& $-0.152^{* * *}$ & \\
Male & $(0.007)$ & \\
& $-0.395^{* * *}$ & \\
Different Location & $0.116^{* * *}$ & \\
& $(0.003)$ & \\
lnExperience & & $0.050^{* * *}$ \\
Entity Type & & $(0.003)$ \\
State \& Month FE & $\mathrm{Y}$ & $-0.368^{* * *}$ \\
Primary Diagnosis & $\mathrm{Y}$ & $(0.005)$ \\
Covid Cases & $\mathrm{Y}$ & $\mathrm{Y}$ \\
State Policy & $\mathrm{Y}$ & $\mathrm{Y}$ \\
\hline Observations & $3,227,719$ & $\mathrm{Y}$ \\
Pseudo R-Squared & 0.205 & $0.024,997$ \\
\hline \multirow{2}{*}{$p<0.1,{ }^{* *} p<0.05,{ }^{* * *} p<0.01$} & \\
\end{tabular}

From the provider side, we consider how the experience and organizational affiliation affect one's telehealth adoption. Similarly, we perform a logistic regression using visit-level data from patients who are telehealth adopters. Within this sample, given a patient's willingness to use telehealth, we can pin down factors that affect providers' choices of the visit modality. The 
results are shown in column 2 of Table 6 We find that more experienced providers are more likely to adopt telehealth. Interestingly, physicians affiliated with an organization are less likely to adopt telehealth than independent practitioners, probably because the latter have more flexibility in setting their practice patterns. For both analyses, we also account for the clinical classification code of a patient's primary diagnosis, which may directly affect one's telehealth utilization.

\subsection{Effect of Telehealth Adoption on Covid-19 Infection}

Besides ensuring patients get the health care they need, telehealth intends to promote social distancing. To examine whether telehealth adoption reduces risks to Covid-19 exposure, we refer to the individual data and investigate the correlation between telehealth adoption and the likelihood of Covid-19 infection. For the following analysis, we subset the visit-level data after the telehealth expansion, when telehealth became an available option to patients in our sample. We identify a patient's Covid-19 infection status using one's primary diagnosis code. In particular, a visit with diagnosis codes of U07. 1 or U07. 2 indicates an infection.

For an individual $i$ at time $t$, we create two time series: (1) the visit modality of individual $i$ at time $t$ (denoted by TelehealthVisit $t_{i, t} \in\{0,1\}$ ); (2) whether the individual $i$ is infected with Covid-19 at time $t$

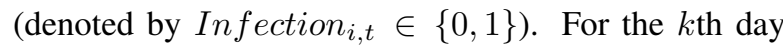
forward (i.e., day $t+k$ ), we then use all individual observations to calculate the average infection rate as the percentage of infected patients, conditional on the visit modality at day $t$ (i.e., $\operatorname{Pr}\left[\right.$ Infection $_{t+k}=$ $1 \mid$ TelehealthVisit $\left._{t}=0\right]$ and $\operatorname{Pr}\left[\right.$ Infection $_{t+k}=$ $1 \mid$ TelehealthVisit $\left._{t}=1\right]$ ).

Table 7 reports the results, where we re-scale the infection rate by a factor of $1 / 10,000$ to allow for more non-zero digits in the statistics. We can see patients who adopted telehealth indeed had a much lower infection rate than those who went through in-person visits. The t-test results suggest that the average infection rates are indeed significantly different between the two visit modalities. Moreover, the difference in the infection rate following these two visit modalities seems to become more salient over time. Although this is just a non-parametric analysis, and we are not arguing for a causal story, the results here at least suggest the potential of telehealth in preventing Covid-19 spread. As a result, we consider telehealth a preferred way of accessing care than in-person visits during the pandemic.
Table 7. Telehealth and Covid-19 Infection

\begin{tabular}{lcccc}
\hline \multicolumn{5}{c}{ Infection Rate } \\
\cline { 2 - 5 } & In-person & Telehealth & Difference & t statistic \\
\hline $\mathrm{t}+2$ & 0.944 & 0.429 & $-0.515^{*} * *$ & 6.202 \\
$\mathrm{t}+4$ & 1.544 & 0.835 & $-0.709^{*} * *$ & 6.533 \\
$\mathrm{t}+6$ & 1.994 & 1.304 & $-0.690^{*} * *$ & 5.454 \\
$\mathrm{t}+8$ & 2.407 & 1.642 & $-0.765^{*} * *$ & 5.467 \\
$\mathrm{t}+10$ & 2.653 & 1.789 & $-0.864^{*} * *$ & 5.888 \\
$\mathrm{t}+12$ & 2.905 & 2.009 & $-0.896^{*} * *$ & 5.815 \\
$\mathrm{t}+14$ & 3.154 & 2.167 & $-0.987^{*} * *$ & 6.153 \\
\hline
\end{tabular}

${ }^{*} p<0.1,{ }^{* *} p<0.05,{ }^{* * *} p<0.01$

\section{Conclusion}

Based on a unique dataset with detailed visit-level information, we evaluated the impact of telehealth policy on care access disparities between rural and urban areas. Contrary to the well-intention of extending patients' swift access to care during the pandemic, telehealth expansion disproportionately benefits urban patients more than rural patients. Robust empirical results suggest that the total patient visits evolved similarly in rural and urban areas, thereby rural-urban disparities in total access to care did not change due to telehealth expansion. Moreover, rural patients have $12.5 \%$ more office visits than urban patients following the telehealth expansion. In contrast, telehealth visits in rural areas are much lower, with rural patients having $158.3 \%$ fewer telehealth visits than urban patients. We also find that the utilization of telehealth associates with a lower infection rate. Overall, the results imply that urban patients benefit from the expansion by having significantly more telehealth visits and fewer in-person visits, while rural patients continue with office visits and thus are potentially at higher risks of infections. Through individual-level analysis, we further examine several barriers to telehealth adoption. We find that social determinants (i.e., geographic location, age, gender, travel distance, providers' organizational affiliation) significantly correlate with one's choice of visiting modality.

Our paper adds to the literature on rural-urban disparity, a significant and long-standing social issue, especially within the healthcare context. Our results also contribute to the nascent literature on health IT and telehealth in particular. Using the telehealth expansion as a shock, we identify how patients of different social-economic statuses reacted to the telehealth availability and how this affects the rural-urban disparity regarding the channels of access to care. Our findings indicate that, despite the overall increase in telehealth usage following the telehealth extension, 
rural patients are significantly disadvantaged and have a much lower adoption rate of this safe option than their urban counterparts. We hope that our findings could increase the awareness of the issue. For healthcare decision-makers, they may consider taking further actions to clean up the roadblocks to "truly" increase rural patients' access to telehealth. As the Covid-19 pandemic accelerated the trend toward remote health, policymakers shall take this opportunity and bear in mind the issue of rural-urban disparity when implementing the policy change.

\section{References}

[1] E. R. Dorsey and E. J. Topol, "State of telehealth," New England Journal of Medicine, vol. 375, no. 2, pp. 154-161, 2016.

[2] J. E. Hollander and B. G. Carr, "Virtually perfect? Telemedicine for COVID-19," New England Journal of Medicine, vol. 382, no. 18, pp. 1679-1681, 2020.

[3] R. Cui, H. Ding, and F. Zhu, "Gender inequality in research productivity during the COVID-19 pandemic," Manufacturing \& Service Operations Management, 2021.

[4] E. R. Dorsey, V. Venkataraman, M. J. Grana, M. T. Bull, B. P. George, C. M. Boyd, C. A. Beck, B. Rajan, A. Seidmann, and K. M. Biglan, "Randomized controlled clinical trial of "virtual house calls" for parkinson disease," JAMA Neurology, vol. 70, no. 5, pp. 565-570, 2013.

[5] H. Bavafa, L. M. Hitt, and C. Terwiesch, "The impact of e-visits on visit frequencies and patient health: Evidence from primary care," Management Science, vol. 64, no. 12 , pp. 5461-5480, 2018.

[6] K. Delana, S. Deo, K. Ramdas, G. Babu, and T. Ravilla, "Multichannel delivery in healthcare: the impact of telemedicine centers in southern India," Indian School of Business, 2019.

[7] S. Saghafian, W. J. Hopp, S. M. Iravani, Y. Cheng, and D. Diermeier, "Workload management in telemedical physician triage and other knowledge-based service systems," Management Science, vol. 64, no. 11, pp. 5180-5197, 2018.

[8] Ö. E. Çakıcı and A. F. Mills, "On the role of teletriage in healthcare demand management," Manufacturing \& Service Operations Management, 2020.

[9] B. Rajan, T. Tezcan, and A. Seidmann, "Service systems with heterogeneous customers: Investigating the effect of telemedicine on chronic care," Management Science, vol. 65, no. 3, pp. 1236-1267, 2019.

[10] S. Sun, S. F. Lu, and H. Rui, "Does telemedicine reduce emergency room congestion? Evidence from new york state," Information Systems Research, vol. 31, no. 3, pp. 972-986, 2020.

[11] S. A. Cohen, S. K. Cook, T. A. Sando, and N. J. Sabik, "What aspects of rural life contribute to rural-urban health disparities in older adults? evidence from a national survey," The Journal of Rural Health, vol. 34 no. 3, pp. 293-303, 2018.
[12] M. E. Johnson, C. Brems, T. D. Warner, and L. W. Roberts, "Rural-urban health care provider disparities in alaska and new mexico," Administration and Policy in Mental Health and Mental Health Services Research, vol. 33, no. 4, pp. 504-507, 2006.

[13] M. Liu, Q. Zhang, M. Lu, C.-S. Kwon, and H. Quan, "Rural and urban disparity in health services utilization in china," Medical Care, pp. 767-774, 2007.

[14] J. Masuda, M. Kishi, N. Kumagai, T. Yamazaki, K. Sakata, T. Higuma, A. Ogimoto, K. Dohi, T. Tanigawa, H. Hanada, et al., "Rural-urban disparity in emergency care for acute myocardial infarction in japan," Circulation Journal, pp. CJ-17, 2018.

[15] J. M. Goh, G. Gao, and R. Agarwal, "The creation of social value: Can an online health community reduce rural-urban health disparities?," MIS Quarterly, vol. 40, no. $1,2016$.

[16] M. J. Lyerly, T.-C. Wu, M. T. Mullen, K. C. Albright, C. Wolff, A. K. Boehme, C. C. Branas, J. C. Grotta, S. I. Savitz, and B. G. Carr, "The effects of telemedicine on racial and ethnic disparities in access to acute stroke care," Journal of Telemedicine and Telecare, vol. 22, no. 2, pp. 114-120, 2016.

[17] S. Khairat, T. Haithcoat, S. Liu, T. Zaman, B. Edson, R. Gianforcaro, and C.-R. Shyu, "Advancing health equity and access using telemedicine: A geospatial assessment," Journal of the American Medical Informatics Association, vol. 26, no. 8-9, pp. 796-805, 2019.

[18] R. Chunara, Y. Zhao, J. Chen, K. Lawrence, P. A. Testa, O. Nov, and D. M. Mann, "Telemedicine and healthcare disparities: A cohort study in a large healthcare system in new york city during COVID-19," Journal of the American Medical Informatics Association, vol. 28, no. 1, pp. 33-41, 2021.

[19] R. Seamans and F. Zhu, "Responses to entry in multi-sided markets: The impact of Craigslist on local newspapers," Management Science, vol. 60, no. 2, pp. 476-493, 2014.

[20] M. Lechner, N. Rodriguez-Planas, and D. Fernández Kranz, "Difference-in-difference estimation by fe and ols when there is panel non-response," Journal of Applied Statistics, vol. 43, no. 11, pp. 2044-2052, 2016.

[21] G. Wang and T. Dai, "The spillover effect of suspending non-essential surgery: Evidence from kidney transplantation," Available at SSRN 3719662, 2020.

[22] P. R. Rosenbaum and D. B. Rubin, "The central role of the propensity score in observational studies for causal effects," Biometrika, vol. 70, no. 1, pp. 41-55, 1983.

[23] E. A. Stuart, "Matching methods for causal inference: A review and a look forward," Statistical Science: A Review Journal of the Institute of Mathematical Statistics, vol. 25, no. 1, p. 1, 2010. 